\title{
Stability of equilibria for population games with uncertain parameters under bounded rationality
}

\author{
Wei Zhao ${ }^{1}$, Hui Yang ${ }^{1 *}$ (D) Xicai Deng ${ }^{2}$ and Chongyi Zhong ${ }^{2}$
}

"Correspondence:

hui-yang@163.com

${ }^{1}$ School of Mathematics and

Statistics, Guizhou University,

550025, Guiyang, Guizhou, China

Full list of author information is

available at the end of the article

\section{Springer}

\begin{abstract}
Under the assumption that the range of varying uncertain parameters is known, some results of existence and stability of equilibria for population games with uncertain parameters are investigated in this paper. On the basis of NS equilibria in classical noncooperative games, the concept of NS equilibria for population games with uncertain parameters is defined. Using some hypotheses about the continuity and convexity of payoff functions, the existence of NS equilibria in population games is also proved by Fan-Glicksberg fixed point theorem. Furthermore, we establish a bounded rationality model of population games with uncertain parameters, and draw the conclusions about the stability of NS equilibrium in this model by constructing the rationality function and studying its properties.
\end{abstract}

MSC: 90C31; 91A10; 91A26; 91A44

Keywords: Population games; Uncertain parameters; NS equilibrium; Existence; Stability; Bounded rationality

\section{Introduction}

In real life, there are many games concerning strategic interactions among numerous individuals. Population game theory is a powerful tool in the context of competitions with large numbers of individuals and is widely applied to various fields, such as biology, traffic, sociology, science, technology and so on. The classical population game theory stems from "mass-action" explanation of equilibrium points in the doctoral dissertation [1] of Nash and it has been further studied by many game theorists [2-6]. The monograph [3] of Sandholm published in 2011 is one of the most representative works, which systematically elaborated the theory and applications of population games and their evolutionary dynamics, and provided an important foundation for the subsequent research of population games. In recent years, Yang et al. [7, 8] extended single-objective population games to multiobjective ones. They proposed the concepts of weighted Nash equilibria, ParetoNash equilibria and weakly Pareto-Nash equilibria for multiobjective population games, then proved the existence of equilibria and studied the stability of equilibrium points by nonlinear analysis methods. Yang and Zhang [9] first introduced the notion of coopera-

(c) The Author(s) 2021. This article is licensed under a Creative Commons Attribution 4.0 International License, which permits use sharing, adaptation, distribution and reproduction in any medium or format, as long as you give appropriate credit to the original author(s) and the source, provide a link to the Creative Commons licence, and indicate if changes were made. The images or other third party material in this article are included in the article's Creative Commons licence, unless indicated otherwise in a credit line to the material. If material is not included in the article's Creative Commons licence and your intended use is not permitted by statutory regulation or exceeds the permitted use, you will need to obtain permission directly from the copyright holder. To view a copy of this licence, visit http://creativecommons.org/licenses/by/4.0/. 
tive equilibria for population games and analyzed the existence and essential stability of the relevant equilibrium points.

Most existing economic models are always based on the assumption of complete rationality, that is to say, every decision maker can maximize his/her own interests under some constraints. However, people cannot have perfect thinking ability, therefore the assumption of complete rationality is too ideal, which limits the application of the models. Thus, it is necessary to introduce bounded rationality into the economic models. Anderlini and Canning [10] established a model of game with abstract bounded rationality functions. They regarded the economic problems satisfying some certain conditions as an abstract space, and provided the definitions and conditions of structural stability and robustness. Considering the assumptions of their model are too demanding and many economic models fail to meet them, Yu et al. [11-14] weakened these conditions, extended the application of the model to cover other non-linear problems, and finally made some new and profound conclusions. As for population games, in which individuals are always bounded rational, it is thereby necessary to discuss the bounded rationality in population games.

All the above studies are based on the deterministic theory. However, due to the incompleteness of information, non-complete rationality of behaviors, or the restriction and influence of uncertain factors such as climate and environment in the real world, the decision-making environment is often uncertain. As a result, we need to introduce the uncertain parameters to describe such mathematical models. When the influence of uncertain parameters is small, it can be ignored, then a satisfactory approximate solution can be figured out. But when the influence cannot be ignored, its negligence may give an erroneous solution, so it is of great importance to deal with these uncertain parameters in such a case. Providing some characteristics of uncertain parameters such as randomness, fuzziness or fuzzy randomness, the related problems can be investigated by using Bayesian game [15], fuzzy game [16] or fuzzy Bayesian game [17]. Nevertheless, if the above characteristics are unknown and the participants can only predict the change range of uncertain parameters, these parameters need to be dealt with in different ways. By combining the concepts of classic Nash equilibrium and weak Pareto (or Slater) efficient solution for multiobjective optimization, Zhukovskii [18] first put forward to the notion of Nash-Slater (or NS for short)equilibrium for an n-person noncooperative game with uncertain parameters, and then studied the existence of NS equilibrium. On this basis, many scholars have conducted a large number of studies to investigate various problems of games with uncertain parameters [19-21].

In this paper, inspired by the study so far, we introduce the uncertain parameters for population games, propose the concept of NS equilibrium for population games with uncertain parameters, and prove the existence of NS equilibrium by using Fan-Glicksberg fixed point theorem. Moreover, through introducing an abstract rationality function, we establish a bounded rationality model for population games with uncertain parameters, and study the stability of NS equilibria in this model.

\section{Preliminaries}

We first recall some elementary definitions and conclusions, which we will mainly use in the following sections.

Definition 2.1 ([22]) Let $E$ and $H$ be two topological vector spaces, $X$ be a nonempty convex set in $E$ and $C$ be a convex cone in $H$ with int $C \neq \emptyset$. A vector-valued function 
$f$ from $X$ to $H$ is called to be $C$-quasiconvex-like if for any $x_{1}, x_{2} \in X$ and $\lambda \in(0,1)$, it satisfies $f\left(x_{1}\right) \in f\left(\lambda x_{1}+(1-\lambda) x_{2}\right)+C$ or $f\left(x_{2}\right) \in f\left(\lambda x_{1}+(1-\lambda) x_{2}\right)+C . f$ is called to be $C$-quasiconcave-like if $-f$ is $C$-quasiconvex-like.

Remark 2.1 If $H=R, C=[0,+\infty)$, then $f$ is $C$-quasiconvex-like (or $C$-quasiconcave-like) is equivalent to $f$ is quasiconvex (or quasiconcave). Therefore $C$-quasiconvex-like (or $C$ quasiconcave-like) is an extension of quasiconvex (or quasiconcave).

Lemma 2.1 ([23]) Let $X$ and $Y$ be two Hausdorff topological spaces. Assume that $S: X \rightarrow$ $2^{Y}$ is closed and $Y$ is compact, then $S$ is upper semicontinuous on $X$.

Lemma 2.2 ([24, 25] Fan-Glicksberg fixed point theorem) Let $X$ be a nonempty compact convex subset of a locally convex Hausdorff topological vector space E. IfS :X $\rightarrow 2^{X}$ is upper semicontinuous on $X$, and $S(x)$ is a nonempty compact convex set for every $x \in X$, then there is $\bar{x} \in X$ such that $\bar{x} \in S(\bar{x})$.

Lemma 2.3 ([11]) Let $X, Y$ be two metric spaces, $K(X)$ be a set containing all nonempty compact sets in $X, B_{m}, B \in K(X), y_{m}, y \in Y$ and $p^{m}, p$ be continuous functions defined on $X \times Y, m=1,2, \ldots$ If $h\left(B_{m}, B\right) \rightarrow 0$, where $h$ is the Hausdorff distance defined on $X, y_{m} \rightarrow y$ and $\sup _{(x, y) \in X \times Y}\left|p^{m}(x, y)-p(x, y)\right| \rightarrow 0$, then $\max _{v \in B_{m}} p^{m}\left(v, y_{m}\right) \rightarrow \max _{v \in B} p(v, y)$.

Lemma 2.4 ([12]) Let $X, Y$ be two metric spaces, $Z$ be a compact metric space, $K(X)$ be a set containing all nonempty compact sets in $X, B_{m}, B \in K(X), y_{m}, y \in Y$ and $p^{m}$, $p$ be continuous functions defined on $X \times Y \times Z, m=1,2, \ldots$. If $h\left(B_{m}, B\right) \rightarrow 0$, where $h$ is the Hausdorff distance defined on $X, y_{m} \rightarrow y$ and $\sup _{(x, y, z) \in X \times Y \times Z}\left|p^{m}(x, y, z)-p(x, y, z)\right| \rightarrow 0$, then

$$
\max _{v \in B_{m}} \min _{z \in Z} p^{m}\left(v, y_{m}, z\right) \rightarrow \max _{v \in B} \min _{z \in Z} p(v, y, z) .
$$

For the following two lemmas, one can refer to [26].

Lemma 2.5 Assume that $X$ and $Y$ are two Hausdorff topological vector spaces, a vectorvalued function $S: X \times Y \rightarrow R^{k}$ is continuous, a set-valued mapping $G: Y \rightarrow 2^{X}$ is continuous on $Y$ with $G(y)$ being a nonempty compact subset of $X$ for any $y \in Y$, and $V(y)=\left\{x \in G(y) \mid S(u, y)-S(x, y) \notin \operatorname{int} R_{+}^{k}\right.$, for any $\left.u \in G(y)\right\}$, then $V(y)$ is a nonempty compact set, and $V: Y \rightarrow 2^{X}$ is upper semicontinuous on $Y$.

Lemma 2.6 If $X$ is a complete metric space and $A$ is a nonempty closed subset of $X$, then the subspace $A$ is complete.

\section{Existence of NS equilibria for population games with uncertain parameters}

Let us recall the concept of NS equilibria for $n$-person noncooperative games with uncertain parameters, see [18].

We denote an $n$-person game with uncertain parameters by $G=(I, X, Y, f(x, y))$. Specifically, $I=\{1, \ldots, n\}$ is the set of players, where $n \geq 1 ; X=\prod_{i \in I} X_{i}$ and $X_{i}$ is the set of strategies of the $i$ th player, where $i=1, \ldots n$; $Y$ is the set of uncertain parameters, $Y \subset R^{m}, m \geq 1$ and $y \in Y$ is a vector of uncertain parameters; $f=\left(f_{1}, \ldots, f_{n}\right), f_{i}: X \times Y \rightarrow R$ is the payoff function of the $i$ th player. For every $i \in I$, denote $x_{\hat{i}}=\left(x_{1}, \ldots, x_{i-1}, x_{i+1}, \ldots, x_{n}\right) \in X_{\hat{i}}=$ $\prod_{j \in I \backslash\{i\}} X_{j}$. 
In this game, suppose that all players only know the range $Y$ of the uncertain parameter vector. Every player selects a strategy to maximize his/her own payoff, meanwhile, the payoff is also a function of uncertain parameters. The game can be described as: we can obtain the strategy profile $x=\left(x_{i}, x_{\hat{i}}\right) \in X$ while each player has selected his/her strategy, if $y \in Y$ is the uncertain parameter vector, then the $i$ th player's payoff is $f_{i}(x, y)$.

Definition 3.1 A pair $\left(x^{*}, y^{*}\right) \in X \times Y$ is an NS equilibrium of $G$ if it satisfies:

(1) $\forall i \in I, f_{i}\left(x_{i}^{*}, x_{\hat{i}}^{*}, y^{*}\right) \geq f_{i}\left(x_{i}, x_{\hat{i}}^{*}, y^{*}\right)$; and

(2) $\forall y \in Y, f\left(x^{*}, y^{*}\right)-f\left(x^{*}, y\right) \notin \operatorname{int} R_{+}^{n}$, where $f=\left(f_{1}, \ldots, f_{n}\right)$ and $\operatorname{int} R_{+}^{n}=\left\{\left(u_{1}, \ldots, u_{n}\right) \in R^{n} \mid u_{i}>0, i=1, \ldots, n\right\}$.

\section{Remark 3.1}

(1) The first condition shows that $x^{*}$ is a Nash equilibrium of the classical noncooperative game $\left(I, X, f\left(\cdot, y^{*}\right)\right)$ given an uncertain parameter vector $y=y^{*}$.

(2) The second condition implies that $y^{*}$ is a Slater optimal (also known as weak Pareto optimal) solution of the multiobjective minimization problem $\min _{y \in Y} f\left(x^{*}, y\right)$ with fixed $x=x^{*}$. In this case, every player holds pessimistic or conservative attitude towards uncertain parameters, that is to say, the payoffs of $n$ players can be viewed as $n$ objectives, we cannot make all $n$ objectives strictly poor under the effect of uncertain parameters.

(3) If $Y=\emptyset$ or $y$ is fixed, the solutions satisfying Definition 3.1 are Nash equilibria for determined classical noncooperative games.

Sandholm [3] provided a detailed description about the concepts of population games and their Nash equilibria. Based on it, we introduce the uncertain parameters into population games and then define the concept of NS equilibria for population games with uncertain parameters.

Suppose that a society containing $N \geq 1$ populations is $\mathcal{P}=\{1,2, \ldots, N\}$. Each population $p \in \mathcal{P}$ consists of sufficient but finite agents with the same strategy set and payoff function. The pure strategy set for agents in population $p$ is presented as $S^{p}=\left\{1,2, \ldots, n^{p}\right\}$, the total number of pure strategies for all populations is $n=\sum_{p \in \mathcal{P}} n^{p}$. The population states of population $p$ is denoted as $X^{p}=\left\{x^{p}=\left(x_{1}^{p}, x_{2}^{p}, \ldots, x_{n^{p}}^{p}\right) \in R_{+}^{n^{p}} \mid \sum_{i=1}^{n^{p}} x_{i}^{p}=1\right\}$, where each scalar $x_{i}^{p}$ of $x^{p}$ indicates the share of agents in population $p$ who select strategy $i \in S^{p}$, and $x^{p}$ is the state (vector) of population $p$. Denoted by $X=\prod_{p \in \mathcal{P}} X^{p}=$ $\left\{x=\left(x^{1}, x^{2}, \ldots, x^{N}\right) \in R_{+}^{n} \mid x^{p} \in X^{p}\right\}$ the social state set, which represents the aggregate behavior in the society.

The model of population games with uncertain parameters is denoted by $\Gamma=\left(X^{p}, F^{p}\right.$, $Y)_{p \in \mathcal{P}} . Y$ is the set of uncertain parameters, $Y \subset R^{m} . F_{i}^{p}: X \times Y \rightarrow R$ indicates the payoff of agents selecting strategy $i \in S^{p}$ in population $p, F^{p}=\left(F_{1}^{p}, F_{2}^{p}, \ldots, F_{n^{p}}^{p}\right): X \times Y \rightarrow R^{n^{p}}$ represents the payoff functions for all strategies in $S^{p}$ and $F=\left(F^{1}, F^{2}, \ldots, F^{N}\right): X \times Y \rightarrow R^{n}$ is the payoff functions of all populations in the society.

Remark 3.2 $X^{p}$ is a simplex in $R^{n^{p}}$, so it is obviously a nonempty convex compact set.

We propose the notion of NS equilibria for $\Gamma$ as follows.

Definition 3.2 A pair $(\tilde{x}, \tilde{y}) \in X \times Y$ is an NS equilibrium of the model $\Gamma$ if it satisfies: 
(1) $\tilde{x}_{i}^{p}>0 \Rightarrow F_{i}^{p}(\tilde{x}, \tilde{y}) \geq F_{j}^{p}(\tilde{x}, \tilde{y}), \forall p \in \mathcal{P}, \forall i, j \in S^{p}$; and

(2) $F^{p}(\tilde{x}, \tilde{y})-F^{p}(\tilde{x}, y) \notin \operatorname{int} R_{+}^{n^{p}}, \forall p \in \mathcal{P}, \forall y \in Y$.

\section{Remark 3.3}

(1) The first condition shows that $\tilde{x}$ is a classical Nash equilibrium for the population game $(X, F(\cdot, \tilde{y}))$ given an uncertain parameter vector $y=\tilde{y}$ (see [3]).

(2) The second condition implies that $\tilde{y}$ is a Slater optimal (also known as weak Pareto optimal) solution of the multiobjective minimization problem $\min _{y \in Y} F(\tilde{x}, y)$ with fixed social state $x=\tilde{x}$. In this case, every individual in all populations holds pessimistic or conservative attitude towards uncertain parameters, that is to say, the payoffs of $n^{p}$ pure strategies chosen by individuals in population $p$ may be viewed as $n^{p}$ objectives, we cannot make all $n^{p}$ objectives strictly poor under the effect of uncertain parameters.

(3) If $Y=\emptyset$ or $y$ is fixed, the solutions satisfying Definition 3.2 are Nash equilibria of determined population games.

Sandholm established an equivalent description of Nash equilibria for population games in [3]. Similarly, two equivalent conditions are easily obtained in population games with uncertain parameters.

Lemma 3.1 Two equivalent conditions are given as following:

(1) $\tilde{x}_{i}^{p}>0 \Rightarrow F_{i}^{p}(\tilde{x}, \tilde{y}) \geq F_{j}^{p}(\tilde{x}, \tilde{y}), \forall p \in \mathcal{P}, \forall i, j \in S^{p}$; and

(2) $\sum_{i \in S^{p}} \tilde{x}_{i}^{p} F_{i}^{p}(\tilde{x}, \tilde{y})=\max _{u^{p} \in X^{p}} \sum_{i \in S^{p}} u_{i}^{p} F_{i}^{p}(\tilde{x}, \tilde{y}), \forall p \in \mathcal{P}$.

Next, we use Fan-Glicksberg fixed point theorem to prove the existence of NS equilibria for population games with uncertain parameters.

Theorem 3.1 Let $\Gamma=\left(X^{p}, F^{p}, Y\right)_{p \in \mathcal{P}}$ be a population game with uncertain parameters, where $Y$ is a nonempty convex compact subset of $R^{m}$. If the payoff functions satisfy the following conditions:

(1) for each $p \in \mathcal{P}$ and $i \in S^{p}, F_{i}^{p}: X \times Y \rightarrow R$ is continuous,

(2) for each $x \in X, y \mapsto F^{p}(x, y)$ is $R_{+}^{n^{p}}$-quasiconvex-like, then there exists at least one NS equilibrium of $\Gamma$.

Proof For each $p \in \mathcal{P}$, a set-valued mapping $T^{p}: X \times Y \rightarrow 2^{X^{p}}$ is given by

$$
T^{p}(x, y)=\left\{z^{p} \in X^{p} \mid \sum_{i \in S^{p}} z_{i}^{p} F_{i}^{p}(x, y)=\max _{u^{p} \in X^{p}} \sum_{i \in S^{p}} u_{i}^{p} F_{i}^{p}(x, y)\right\}
$$

then we obtain $T: X \times Y \rightarrow 2^{X}$ by $T(x, y)=\prod_{p \in \mathcal{P}} T^{p}(x, y)$.

Meanwhile, a set-valued mapping $H^{p}: X \rightarrow 2^{Y}$ is given by

$$
H^{p}(x)=\left\{y \in Y \mid F^{p}(x, y)-F^{p}(x, w) \notin \operatorname{int} R_{+}^{n^{p}}, \forall w \in Y\right\},
$$

then we obtain $H: X \rightarrow 2^{Y}$ by $H(x)=\prod_{p \in \mathcal{P}} H^{p}(x)$.

For each $p \in \mathcal{P}$, it is clear that $T^{p}(x, y)$ is a nonempty compact convex set for every $(x, y) \in X \times Y$. Next, we show that $T^{p}$ is upper semicontinuous. From Lemma 2.1, we only 
need to show the graph of $T^{p}$ is closed. Namely, let $\left\{\left(x^{m}, y^{m}\right)\right\}_{m=1}^{\infty} \subset X \times Y$ be any sequence with $\left(x^{m}, y^{m}\right) \rightarrow(x, y)$, for each $z^{p, m} \in T^{p}\left(x^{m}, y^{m}\right)$ with $z^{p, m} \rightarrow z^{p}$, it needs to verify $z^{p} \in$ $T^{p}(x, y)$. Since $z^{p, m} \in T^{p}\left(x^{m}, y^{m}\right)$, then $\sum_{i \in S^{p}} z^{p, m} F_{i}^{p}\left(x^{m}, y^{m}\right)=\max _{u^{p} \in X^{p}} \sum_{i \in S^{p}} u_{i}^{p} F_{i}^{p}\left(x^{m}, y^{m}\right)$. Furthermore, as $F_{i}^{p}$ is continuous, we get $\sum_{i \in S^{p}} z^{p, m} F_{i}^{p}\left(x^{m}, y^{m}\right) \rightarrow \sum_{i \in S^{p}} z_{i}^{p} F_{i}^{p}(x, y)$ and $\max _{u^{p} \in X^{p}} \sum_{i \in S^{p}} u_{i}^{p} F_{i}^{p}\left(x^{m}, y^{m}\right) \rightarrow \max _{u^{p} \in X^{p}} \sum_{i \in S^{p}} u_{i}^{p} F_{i}^{p}(x, y)$ by Lemma 2.3. It then follows that $\sum_{i \in S^{p}} z_{i}^{p} F_{i}^{p}(x, y)=\max _{u^{p} \in X^{p}} \sum_{i \in S^{p}} u_{i}^{p} F_{i}^{p}(x, y)$, thus we have $z^{p} \in T^{p}(x, y)$.

To sum up, $T^{p}$ is upper semicontinuous and $T^{p}(x, y)$ is a nonempty compact convex set. Hence, $T=\prod_{p \in \mathcal{P}} T^{p}$ is upper semicontinuous and $T(x, y)$ is a nonempty compact convex set.

On the other hand, for each $p \in \mathcal{P}$, we can easily obtain $H^{p}$ is upper semicontinuous with a nonempty compact set $H^{p}(x)$ by Lemma 2.5. We next show $H^{p}(x)$ is a convex set for any $x \in X$. For any $y_{1}, y_{2} \in H^{p}(x)$ and $\lambda \in(0,1)$, suppose that $\lambda y_{1}+(1-\lambda) y_{2} \notin H^{p}(x)$, then there is $w_{0} \in Y$ such that $F^{p}\left(x, \lambda y_{1}+(1-\lambda) y_{2}\right)-F^{p}\left(x, w_{0}\right) \in \operatorname{int} R_{+}^{n^{p}}$. From condition (2), we get $F^{p}\left(x, y_{1}\right) \in F^{p}\left(x, \lambda y_{1}+(1-\lambda) y_{2}\right)+R_{+}^{n^{p}}$, then

$$
\begin{aligned}
& F^{p}\left(x, y_{1}\right)-F^{p}\left(x, w_{0}\right) \\
& \quad=F^{p}\left(x, y_{1}\right)-F^{p}\left(x, \lambda y_{1}+(1-\lambda) y_{2}\right)+F^{p}\left(x, \lambda y_{1}+(1-\lambda) y_{2}\right)-F^{p}\left(x, w_{0}\right) \\
& \quad \in R_{+}^{n^{p}}+\operatorname{int} R_{+}^{n^{p}} \subset \operatorname{int} R_{+}^{n^{p}},
\end{aligned}
$$

which contradicts that $y_{1} \in H^{p}(x)$. Consequently, $H^{p}(x)$ is a convex set for every $x \in X$.

To sum up, $H^{p}$ is upper semicontinuous and $H^{p}(x, y)$ is a nonempty compact convex set. Hence, $H=\prod_{p \in \mathcal{P}} H^{p}$ is upper semicontinuous and $H(x, y)$ is a nonempty compact convex set.

We now define $W: X \times Y \rightarrow 2^{X \times Y}$ by $W(x, y)=T(x, y) \times H(x)$. From the discussion above, we know $W$ is upper semicontinuous and $W(x, y)$ is a nonempty compact convex set. From Lemma 2.2, there is $(\tilde{x}, \tilde{y}) \in X \times Y$ such that $(\tilde{x}, \tilde{y}) \in W(\tilde{x}, \tilde{y})$. Hence, for each $p \in \mathcal{P}$, we have $\tilde{x} \in T^{p}(\tilde{x}, \tilde{y})$, then $\sum_{i \in S^{p}} \tilde{x}_{i}^{p} F_{i}^{p}(\tilde{x}, \tilde{y})=\max _{u^{p} \in X^{p}} \sum_{i \in S^{p}} u_{i}^{p} F_{i}^{p}(\tilde{x}, \tilde{y})$ can be obtained. It is equivalent to $\tilde{x}_{i}^{p}>0 \Rightarrow F_{i}^{p}(\tilde{x}, \tilde{y}) \geq F_{j}^{p}(\tilde{x}, \tilde{y}), \forall p \in \mathcal{P}, \forall i, j \in S^{p}$ from Lemma 3.1; also for each $p \in \mathcal{P}$, we obtain $\tilde{y} \in H(\tilde{x})$, this implies that $F^{p}(\tilde{x}, \tilde{y})-F^{p}(\tilde{x}, y) \notin \operatorname{int} R_{+}^{n^{p}}, \forall p \in \mathcal{P}$, $\forall y \in Y$. Consequently, $(\tilde{x}, \tilde{y})$ is an NS equilibrium of $\Gamma$ as it satisfies two conditions of Definition 3.2.

\section{Stability of equilibria for population games with uncertain parameters under bounded rationality}

In this section, let us first recall the model of bounded rationality established by Anderlini and Canning in [10]. We assume that $(X, d)$ is a metric space and $K(X)$ is the set of all nonempty compact subsets of $X$ throughout this section.

An abstract model of bounded rationality is expressed as $M=(\Lambda, X, T, R)$, where $\Lambda$ is a parameter space; $X$ is an action space; $T: \Lambda \times X \rightarrow 2^{X}$ represents the feasibility mapping and its induced further mapping is $f: \Lambda \rightarrow 2^{X}$, and for any $\lambda \in \Lambda, f(\lambda)=$ $\{x \in X: x \in T(\lambda, x)\}$ and $\operatorname{Graph}(f)=\{(\lambda, x) \in \Lambda \times X: x \in f(\lambda)\} ; R: \operatorname{Graph}(f) \rightarrow R^{+}$is a rationality function. For any $\lambda \in \Lambda$ and $\varepsilon \geq 0$, we define the set of $\varepsilon$-equilibria at $\lambda$ as $E(\lambda, \varepsilon)=\{x \in f(\lambda) \mid R(\lambda, x) \leq \varepsilon\}$ (corresponding to the bounded rationality of agents when $\varepsilon>0$ is small sufficiently). In particular, $E(\lambda)=E(\lambda, 0)=\{x \in f(\lambda) \mid R(\lambda, x)=0\}$ is the set of equilibria at $\lambda$, which corresponds to the complete rationality of agents.

$\mathrm{Yu}$ and $\mathrm{Yu}[11]$ gave the following definitions and stability results. 
Definition 4.1 ([11]) The model $M$ is robust to $\varepsilon$-equilibria at $\lambda \in \Lambda$ if for any $\delta>0$, there is an $\bar{\varepsilon}>0$ such that, for any $\varepsilon$ with $0<\varepsilon<\bar{\varepsilon}$ and any $\lambda^{\prime} \in \Lambda$ with $\rho\left(\lambda, \lambda^{\prime}\right)<$ $\bar{\varepsilon}, h\left(E\left(\lambda^{\prime}, \varepsilon\right), E\left(\lambda^{\prime}\right)\right)<\delta$, where $h$ is the Hausdorff distance defined on $X$.

Definition 4.2 ([11]) The model $M$ is structurally stable at $\lambda \in \Lambda$ if the equilibrium mapping $E: \Lambda \rightarrow K(X)$ is continuous at $\lambda \in \Lambda$, namely, $\forall \lambda_{n} \in \Lambda, \lambda_{n} \rightarrow \lambda$, then $h\left(E\left(\lambda_{n}\right), E(\lambda)\right) \rightarrow$ 0 .

Lemma 4.1 ([11]) Suppose that the metric space $\Lambda$ is complete and the metric space $X$ is compact. If $: \Lambda \rightarrow K(X)$ is upper semicontinuous and $R: \operatorname{Graph}(f) \rightarrow R^{+}$is lower semicontinuous, then the following results hold true.

(1) The equilibrium mapping $E: \Lambda \rightarrow K(X)$ is upper semicontinuous.

(2) There is a dense residual subset $Q$ in $\Lambda$ such that $M$ is structurally stable at each $\lambda \in Q$.

(3) $M$ is robust to $\varepsilon$-equilibria at $\lambda \in \Lambda$ and thus robust to $\varepsilon$-equilibria at $\lambda \in Q$, when $M$ is structurally stable at $\lambda \in \Lambda$.

(4) For any $\lambda \in Q, \lambda_{n} \rightarrow \lambda$, and any $\varepsilon_{n} \rightarrow 0$, then $h\left(E\left(\lambda_{n}, \varepsilon_{n}\right), E(\lambda)\right) \rightarrow 0$.

(5) If $E(\lambda)$ is a singleton set for any $\lambda \in \Lambda$, then $M$ is structurally stable and robust to $\varepsilon$-equilibria at $\lambda \in \Lambda$.

Next, we establish a bounded rationality model in population games with uncertain parameters by constructing an abstract rationality function, and then investigate the stability results of the NS equilibrium in our model.

$\Gamma=\left(X^{p}, F^{p}, Y\right)_{p \in \mathcal{P}}$ is the model of population games with uncertain parameters.

Let $\mathcal{F}=\left\{F=\left(F^{1}, F^{2}, \ldots, F^{N}\right) \mid \forall p \in \mathcal{P}, \forall i \in S^{p}, F_{i}^{p}\right.$ is continuous on $X \times Y ; \forall x \in X, y \mapsto$ $F_{i}^{p}(x, y)$ is $R_{+}^{n^{p}}$-quasiconvex-like\}. Denoted by $E_{N S}(F)$ the collection of all NS equilibria of the population game $F \in \mathcal{F}$, it is obviously that $E_{N S}(F) \neq \emptyset$ from Theorem 3.1.

For any $F=\left(F^{1}, F^{2}, \ldots, F^{N}\right), G=\left(G^{1}, G^{2}, \ldots, G^{N}\right) \in \mathcal{F}$, define

$$
\rho(F, G)=\max _{(x, y) \in X \times Y} \sum_{p \in \mathcal{P}} \sum_{i=1}^{n^{p}}\left|F_{i}^{p}(x, y)-G_{i}^{p}(x, y)\right| .
$$

Lemma 4.2 $(\mathcal{F}, \rho)$ is a complete metric space.

Proof Let $B=\left\{F: X \times Y \rightarrow R^{n} \mid \sup _{(x, y) \in X \times Y}\|F(x, y)\|<+\infty\right\}$. For any $F, G \in B$, define $\rho(F, G)=\max _{(x, y) \in X \times Y} \sum_{p \in \mathcal{P}} \sum_{i=1}^{n^{p}}\left|F_{i}^{p}(x, y)-G_{i}^{p}(x, y)\right|$, it is clear that $(B, \rho)$ is a complete metric space. So we need only show that $\mathcal{F}$ is a closed subset of $B$ by Lemma 2.6. That is, let $\left\{F^{m}=\left(F^{1 m}, F^{2 m}, \ldots, F^{N m}\right)\right\}_{m=1}^{\infty}$ be a sequence of $\mathcal{F}$, and $F^{m} \rightarrow F=\left(F^{1}, F^{2}, \ldots, F^{N}\right)$, we prove that $F \in \mathcal{F}$.

For each $p \in \mathcal{P}$ and $i \in S^{p}$, we first show the continuity of $F_{i}^{p}$. Since $F^{m} \rightarrow F$, for any $\varepsilon>0$, there exists a positive integer $M(\varepsilon)$ such that, for any $m \geq M(\varepsilon)$,

$$
\left|F_{i}^{p}(x, y)-F_{i}^{p, m}(x, y)\right| \leq \varepsilon,
$$

for all $(x, y) \in X \times Y$. Fix $m \geq M(\varepsilon)$, then, for any $(x, y) \in X \times Y$, since $F_{i}^{p, m}(x, y)$ is continuous on $X \times Y$, there exists an open neighborhood $U(x, y)$ of $(x, y)$, for any $\left(x^{\prime}, y^{\prime}\right) \in U(x, y)$, 
we have

$$
\left|F_{i}^{p, m}\left(x^{\prime}, y^{\prime}\right)-F_{i}^{p, m}(x, y)\right| \leq \varepsilon
$$

For the above $\left(x^{\prime}, y^{\prime}\right) \in U(x, y)$, from(4.1), we obtain

$$
\left|F_{i}^{p}\left(x^{\prime}, y^{\prime}\right)-F_{i}^{p, m}\left(x^{\prime}, y^{\prime}\right)\right| \leq \varepsilon .
$$

By (4.1) and (4.2),

$$
\left|F_{i}^{p, m}\left(x^{\prime}, y^{\prime}\right)-F_{i}^{p}(x, y)\right| \leq 2 \varepsilon
$$

Furthermore, by (4.3) and (4.4), we derive $\left|F_{i}^{p}\left(x^{\prime}, y^{\prime}\right)-F_{i}^{p}(x, y)\right| \leq 3 \varepsilon$, thus $F_{i}^{p}$ is continuous at $(x, y)$.

Next, it will be proven that $y \mapsto F^{p}(x, y)$ is $R_{+}^{n^{p}}$-quasiconvex-like for any $x \in X$. For every $p \in \mathcal{P}$, consider a sequence $\left\{F^{p, m}=\left(F_{1}^{p, m}, F_{2}^{p, m}, \ldots, F_{n^{p}}^{p, m}\right)\right\}_{m=1}^{\infty}$. Let $V$ be any open neighborhood of zero element $\theta$ in $R_{+}^{n^{p}}$. For any $(x, y) \in X \times Y$, since $F^{p, m} \rightarrow F^{p}$, there is a positive integer $M^{0}$, such that $F^{p, m}(x, y) \in F^{p}(x, y)+\frac{V}{2}$ and $F^{p}(x, y) \in F^{p, m}(x, y)+\frac{V}{2}$ for any $m \geq M^{0}$. Also since $F^{m} \in \mathcal{F}$, then, for any $x \in X, y \rightarrow F^{p, m}(x, y)$ is $R_{+}^{n^{p}}$-quasiconvex-like, i.e., for any $y_{1}, y_{2} \in Y$ and any $\lambda \in(0,1)$,

$$
F^{p, m}\left(x, y_{1}\right) \in F^{p, m}\left(x, \lambda y_{1}+(1-\lambda) y_{2}\right)+R_{+}^{n^{p}},
$$

or

$$
F^{p, m}\left(x, y_{2}\right) \in F^{p, m}\left(x, \lambda y_{1}+(1-\lambda) y_{2}\right)+R_{+}^{n^{p}} .
$$

Thus, fix $m \geq M^{0}$, for each $x \in X$, for any $y_{1}, y_{2} \in Y$ and $\lambda \in(0,1)$,

$$
\begin{aligned}
F^{p}\left(x, y_{1}\right) & \in F^{p, m}\left(x, y_{1}\right)+\frac{V}{2} \\
& \subset F^{p, m}\left(x, \lambda y_{1}+(1-\lambda) y_{2}\right)+\frac{V}{2}+R_{+}^{n^{p}} \\
& \subset F^{p}\left(x, \lambda y_{1}+(1-\lambda) y_{2}\right)+V+R_{+}^{n^{p}},
\end{aligned}
$$

or $F^{p}\left(x, y_{2}\right) \in F^{p}\left(x, \lambda y_{1}+(1-\lambda) y_{2}\right)+V+R_{+}^{n^{p}}$ can be proved similarly. Due to the arbitrariness of $V$, it follows that $F^{p}\left(x, y_{1}\right) \in F^{p}\left(x, \lambda y_{1}+(1-\lambda) y_{2}\right)+R_{+}^{n^{p}}$ or $F^{p}\left(x, y_{2}\right) \in F^{p}\left(x, \lambda y_{1}+(1-\lambda) y_{2}\right)+$ $R_{+}^{n^{p}}$. Namely, for each $x \in X, y \rightarrow F^{p}(x, y)$ is $R_{+}^{n^{p}}$-quasiconvex-like.

To sum up, $\mathcal{F}$ is a closed subset of $B$ and therefore $(\mathcal{F}, \rho)$ is a complete metric space.

Now, we establish the model of bounded rationality for population games with uncertain parameters. Consider the model $M_{1}=(\mathcal{F}, X, Y, T, R): \mathcal{F}$ is the space we mentioned above; $X=\prod_{p \in \mathcal{P}} X^{p}$ is a set of social states; $Y$ is a space of uncertain parameters; $T: \mathcal{F} \times X \times Y \rightarrow$ $2^{X \times Y}$ represents the feasibility mapping and its induced further mapping is $f: \mathcal{F} \rightarrow 2^{X \times Y}$. Define $T(\mathcal{F}, x, y)=X \times Y$, then, for any $F \in \mathcal{F}, f(F)=\{(x, y) \in X \times Y:(x, y) \in T(\mathcal{F}, x, y)\}=$ 
$X \times Y$. Clearly, $f$ is continuous and $f(F)$ is nonempty and compact. Now we define the rationality function $R: \mathcal{F} \times X \times Y \rightarrow R_{+}$as

$$
\begin{aligned}
& R(F, x, y) \\
& \quad=\max _{p \in \mathcal{P}} \max _{u^{p} \in X^{p}} \sum_{i \in S^{p}}\left(u_{i}^{p}-x_{i}^{p}\right) F_{i}^{p}(x, y)+\max _{p \in \mathcal{P}} \max _{v \in Y} \min _{\left\|w^{p}\right\|=1, w^{p} \in R_{+}^{n^{p}}}\left\langle w^{p}, F^{p}(x, y)-F^{p}(x, v)\right\rangle .
\end{aligned}
$$

We will show the following results about the rationality function.

\section{Lemma 4.3}

(1) For any $F \in \mathcal{F}$ and $(x, y) \in X \times Y$, we have $R(F, x, y) \geq 0$.

(2) $R(F, x, y)=0$ if and only if $(x, y) \in E_{N S}(F)$.

Proof (1) For any $F \in \mathcal{F}$ and $(x, y) \in X \times Y$, we obtain

$$
\begin{aligned}
& R(F, x, y) \\
& \quad \geq \max _{p \in \mathcal{P}} \sum_{i \in S^{p}}\left(x_{i}^{p}-x_{i}^{p}\right) F_{i}^{p}(x, y)+\max _{p \in \mathcal{P}} \min _{\left\|w^{p}\right\|=1, w^{p} \in R_{+}^{n^{p}}}\left\langle w^{p}, F^{p}(x, y)-F^{p}(x, y)\right\rangle \\
& \quad=0 .
\end{aligned}
$$

(2) If $R(F, x, y)=0$, then

$$
\begin{aligned}
& \max _{p \in \mathcal{P}} \max _{u^{p} \in X^{p}} \sum_{i \in S^{p}}\left(u_{i}^{p}-x_{i}^{p}\right) F_{i}^{p}(x, y)=0, \\
& \max _{p \in \mathcal{P}} \max _{v \in Y} \min _{\left\|w^{p}\right\|=1, w^{p} \in R_{+}^{p}}\left\langle w^{p}, F^{p}(x, y)-F^{p}(x, v)\right\rangle=0 .
\end{aligned}
$$

By (4.5), for every $p \in \mathcal{P}$ and $u^{p} \in X^{p}$, we can get $\sum_{i \in S^{p}} x_{i}^{p} F_{i}^{p}(x, y)=\max _{u^{p} \in X^{p}} \sum_{i \in S^{p}} u_{i}^{p} F_{i}^{p}(x$, $y$ ) since $\sum_{i \in S^{p}}\left(u_{i}^{p}-x_{i}^{p}\right) F_{i}^{p}(x, y) \leq 0$. Note that it is equivalent to $x_{i}^{p}>0 \Rightarrow F_{i}^{p}(x, y) \geq$ $F_{j}^{p}(x, y), \forall p \in \mathcal{P}, \forall i, j \in S^{p}$ from Lemma 3.1. Thus $(x, y)$ satisfies condition (1) of Definition 3.2.

On the other hand, if there is $v_{0} \in Y$ and $p_{0} \in \mathcal{P}$ such that $F^{p_{0}}(x, y)-F^{p_{0}}\left(x, v_{0}\right) \in$ int $R_{+}^{n^{p}}$. Let $W^{p_{0}}=\left\{w^{p_{0}}:\left\|w^{p_{0}}\right\|=1, w^{p_{0}} \in R_{+}^{n^{p_{0}}}\right\}$, then, for any $w^{p_{0}} \in W^{p_{0}}$, we obtain $\left\langle w^{p_{0}}, F^{p_{0}}(x, y)-F^{p_{0}}\left(x, v_{0}\right)\right\rangle>0$. Since $W^{p_{0}}$ is compact, then

$$
\min _{\left\|w^{p_{0}}\right\|=1, w^{p_{0}} \in R_{+}^{n^{p_{0}}}}\left\langle w^{p_{0}}, F^{p_{0}}(x, y)-F^{p_{0}}\left(x, v_{0}\right)\right\rangle>0 .
$$

Thus

$$
\begin{aligned}
& \max _{p \in \mathcal{P}} \max _{v \in Y} \min _{\left\|w^{p}\right\|=1, w^{p} \in R_{+}^{n^{p}}}\left\langle w^{p}, F^{p}(x, y)-F^{p}(x, v)\right\rangle \\
& \geq \max _{p \in \mathcal{P}} \min _{\left\|w^{p}\right\|=1, w^{p} \in R_{+}^{n^{p}}}\left\langle w^{p}, F^{p}(x, y)-F^{p}\left(x, v_{0}\right)\right\rangle \\
& \geq \min _{\left\|w^{p} 0\right\|=1, w^{p_{0}} \in R_{+}^{n^{p_{0}}}}\left\langle w^{p_{0}}, F^{p_{0}}(x, y)-F^{p_{0}}\left(x, v_{0}\right)\right\rangle
\end{aligned}
$$

$>0$. 
which contradicts (4.6). So for each $p \in \mathcal{P}$ and $v \in Y$, we have $F^{p}(x, y)-F^{p}(x, v) \notin \operatorname{int} R_{+}^{n^{p}}$, then $(x, y)$ satisfies condition (2) of Definition 3.2.

To sum up, $(x, y) \in E_{N S}(F)$.

Conversely, if $(x, y) \in E_{N S}(F)$, from Definition 3.2, we get

$$
\begin{aligned}
& x_{i}^{p}>0 \Rightarrow F_{i}^{p}(x, y) \geq F_{j}^{p}(x, y), \quad \forall p \in \mathcal{P}, \forall i, j \in S^{p}, \\
& F^{p}(x, y)-F^{p}(x, v) \notin \operatorname{int} R_{+}^{n^{p}}, \quad \forall p \in \mathcal{P}, \forall v \in Y .
\end{aligned}
$$

Due to (4.7), we obtain $\sum_{i \in S^{p}} x_{i}^{p} F_{i}^{p}(x, y)=\max _{u^{p} \in X^{p}} \sum_{i \in S^{p}} u_{i}^{p} F_{i}^{p}(x, y)$ for each $p \in \mathcal{P}$ by Lemma 3.1. Consequently, it holds true that $\max _{p \in \mathcal{P}} \max _{u^{p} \in X^{p}} \sum_{i \in S^{p}}\left(u_{i}^{p}-x_{i}^{p}\right) F_{i}^{p}(x, y)=0$.

Furthermore, for any $p \in \mathcal{P}$ and $v \in Y$, let $I(v)=\left\{i: F_{i}^{p}(x, y)-F_{i}^{p}(x, v) \leq 0\right\}$. Clearly, $I(v) \neq$ $\emptyset$ by (4.8). Let $i_{0} \in I(v)$ and define $\stackrel{\underline{w}}{\underline{w}} \in\left\{w^{p} \in R_{+}^{n^{p}}:\left\|w^{p}\right\|=1\right\}$, where $\widehat{w}_{i_{0}}^{p}=1$ and $\hat{w}_{i}^{p}=0(i \neq$ $\left.i_{0}\right)$, then $\left\langle\hat{w}^{p}, F^{p}(x, y)-F^{p}(x, v)\right\rangle=F_{i_{0}}^{p}(x, y)-F_{i_{0}}^{p}(x, v) \leq 0$. Therefore

$$
\min _{\left\|w^{p}\right\|=1, w^{p} \in R_{+}^{n^{p}}}\left\langle w^{p}, F^{p}(x, y)-F^{p}(x, v)\right\rangle \leq 0 .
$$

Thus

$$
\max _{p \in \mathcal{P}} \max _{v \in Y} \min _{\left\|w^{p}\right\|=1, w^{p} \in R_{+}^{n^{p}}}\left\langle w^{p}, F^{p}(x, y)-F^{p}(x, v)\right\rangle \leq 0 .
$$

Similar to the proof of (1), it is easy to see that

$$
\max _{p \in \mathcal{P}} \max _{v \in Y} \min _{\left\|w^{p}\right\|=1, w^{p} \in R_{+}^{n^{p}}}\left\langle w^{p}, F^{p}(x, y)-F^{p}(x, v)\right| \geq 0,
$$

then

$$
\max _{p \in \mathcal{P}} \max _{v \in Y} \min _{\left\|w^{p}\right\|=1, w^{p} \in R_{+}^{n^{p}}}\left\langle w^{p}, F^{p}(x, y)-F^{p}(x, v)\right\rangle=0 .
$$

To sum up, $R(F, x, y)=0$.

Lemma 4.4 $R(F, x, y)$ is continuous on $\mathcal{F} \times X \times Y$.

Proof For any $\left(F^{n}, x^{n}, y^{n}\right) \in \mathcal{F} \times X \times Y$ with $\left(F^{n}, x^{n}, y^{n}\right) \rightarrow(F, x, y)(n \rightarrow+\infty)$, we need to prove that $R\left(F^{n}, x^{n}, y^{n}\right) \rightarrow R(F, x, y)$ as $n \rightarrow+\infty$.

For each $p \in \mathcal{P}$, let

$$
\begin{aligned}
& \varphi^{p}(u, x, y)=\sum_{i \in S^{p}}\left(u_{i}^{p}-x_{i}^{p}\right) F_{i}^{p}(x, y), \\
& \varphi^{p, n}\left(u, x^{n}, y^{n}\right)=\sum_{i \in S^{p}}\left(u_{i}^{p}-x_{i}^{p, n}\right) F_{i}^{p, n}\left(x^{n}, y^{n}\right), \\
& \phi^{p}\left(w^{p}, v, x, y\right)=\left\langle w^{p}, F^{p}(x, y)-F^{p}(x, v)\right\rangle, \\
& \phi^{p, n}\left(w^{p}, v, x^{n}, y^{n}\right)=\left\langle w^{p}, F^{p, n}\left(x^{n}, y^{n}\right)-F^{p, n}\left(x^{n}, v\right)\right\rangle .
\end{aligned}
$$

(1) We first prove that $\max _{p \in \mathcal{P}} \max _{u^{p} \in X^{p}} \varphi^{p, n}\left(u, x^{n}, y^{n}\right) \rightarrow \max _{p \in \mathcal{P}} \max _{u^{p} \in X^{p}} \varphi^{p}(u, x, y)$. 
Since $X$ is compact, for each $p \in \mathcal{P}, X^{p}$ is compact. So there exists $K>0$ such that $\left\|x^{p}\right\| \leq$ $K$ for every $x^{p} \in X^{p}$. As $F^{p, n} \rightarrow F^{p}$, then, for any $\varepsilon_{1}>0$, there is a positive integer $N_{1}$ such that $\left\|F^{p, n}-F^{p}\right\|<\frac{\varepsilon_{1}}{2 K}$ for any $n \geq N_{1}$. Thus, for the above $n$, for each $p \in \mathcal{P}$, we have

$$
\begin{aligned}
& \left|\varphi^{p, n}(u, x, y)-\varphi^{p}(u, x, y)\right| \\
& \quad=\left|\sum_{i \in S^{p}}\left(u_{i}^{p}-x_{i}^{p}\right) F_{i}^{p, n}(x, y)-\sum_{i \in S^{p}}\left(u_{i}^{p}-x_{i}^{p}\right) F_{i}^{p}(x, y)\right| \\
& \quad=\left|\left\langle u^{p}-x^{p}, F^{p, n}(x, y)-F^{p}(x, y)\right\rangle\right| \\
& \quad \leq\left\|u^{p}-x^{p}\right\|\left\|F^{p, n}(x, y)-F^{p}(x, y)\right\| \\
& \quad \leq\left(\left\|u^{p}\right\|+\left\|x^{p}\right\|\right)\left\|F^{p, n}(x, y)-F^{p}(x, y)\right\| \\
& \quad \leq 2 K \cdot \frac{\varepsilon_{1}}{2 K}=\varepsilon_{1} .
\end{aligned}
$$

This shows that $\varphi^{p, n}(u, x, y) \rightarrow \varphi^{p}(u, x, y)$ as $n \rightarrow+\infty$.

It implies that $\max _{u^{p} \in X^{p}} \varphi^{p, n}\left(u, x^{n}, y^{n}\right) \rightarrow \max _{u^{p} \in X^{p}} \varphi^{p}(u, x, y)$ from Lemma 2.3. Hence, we have $\max _{p \in \mathcal{P}} \max _{u^{p} \in X^{p}} \varphi^{p, n}\left(u, x^{n}, y^{n}\right) \rightarrow \max _{p \in \mathcal{P}} \max _{u^{p} \in X^{p}} \varphi^{p}(u, x, y)$.

(2) Next, we show that

$$
\max _{p \in \mathcal{P}} \max _{v \in Y} \min _{\left\|w^{p}\right\|=1, w^{p} \in R_{+}^{n^{p}}} \phi^{p, n}\left(w^{p}, v, x^{n}, y^{n}\right) \rightarrow \max _{p \in \mathcal{P}} \max _{v \in Y} \min _{\left\|w^{p}\right\|=1, w^{p} \in R_{+}^{n^{p}}} \phi^{p}\left(w^{p}, v, x, y\right) .
$$

For every $p \in \mathcal{P}$, as $F^{p, n} \rightarrow F^{p}$, then, for any $\varepsilon_{2}>0$, there exists a positive integer $N_{2}$ such that $\left\|F^{p, n}\left(x^{n}, y^{n}\right)-F^{p}\left(x^{n}, y^{n}\right)\right\|<\frac{\varepsilon_{2}}{4}$ and $\left\|F^{p, n}\left(x^{n}, v\right)-F^{p}\left(x^{n}, v\right)\right\|<\frac{\varepsilon_{2}}{4}$ can be satisfied simultaneously for any $n \geq N_{2}$.

Besides, for each $i \in S^{p}$, since $F_{i}^{p}$ is continuous at $(x, y)$ and $\left(x^{n}, y^{n}\right) \rightarrow(x, y)$, there exists a positive integer $N_{3}$ such that $\left\|F^{p}\left(x^{n}, y^{n}\right)-F^{p}(x, y)\right\|<\frac{\varepsilon_{2}}{4}$ and $\left\|F^{p}\left(x^{n}, v\right)-F^{p}(x, v)\right\|<\frac{\varepsilon_{2}}{4}$ can be satisfied simultaneously for any $n \geq N_{3}$.

Let $N=\max \left\{N_{2}, N_{3}\right\}$, for any $n>N$, we have

$$
\begin{aligned}
&\left|\phi^{p, n}\left(w^{p}, v, x^{n}, y^{n}\right)-\phi^{p}(w, v, x, y)\right| \\
&=\left|\left\langle w^{p}, F^{p, n}\left(x^{n}, y^{n}\right)-F^{p, n}\left(x^{n}, v\right)\right\rangle-\left\langle w^{p}, F^{p}(x, y)-F^{p}(x, v)\right\rangle\right| \\
& \leq \mid\left\langle w^{p}, F^{p, n}\left(x^{n}, y^{n}\right)-F^{p}(x, y) \|+\left|\left\langle w^{p}, F^{p, n}\left(x^{n}, v\right)-F^{p}(x, v)\right\rangle\right|\right. \\
& \leq\left\|w^{p}\right\|\left\|F^{p, n}\left(x^{n}, y^{n}\right)-F^{p}\left(x^{n}, y^{n}\right)\right\|+\left\|w^{p}\right\|\left\|F^{p}\left(x^{n}, y^{n}\right)-F^{p}(x, y)\right\| \\
&+\left\|w^{p}\right\|\left\|F^{p, n}\left(x^{n}, v\right)-F^{p}\left(x^{n}, v\right)\right\|+\left\|w^{p}\right\|\left\|F^{p}\left(x^{n}, v\right)-F^{p}(x, v)\right\| \\
& \leq \frac{\varepsilon_{2}}{4}+\frac{\varepsilon_{2}}{4}+\frac{\varepsilon_{2}}{4}+\frac{\varepsilon_{2}}{4}=\varepsilon_{2} .
\end{aligned}
$$

Therefore, we obtain $\phi^{p, n}\left(w^{p}, v, x^{n}, y^{n}\right) \rightarrow \phi^{p}\left(w^{p}, v, x, y\right)$ for each $p \in \mathcal{P}$. From Lemma 2.4, it follows that

$$
\max _{v \in Y} \min _{\left\|w^{p}\right\|=1, w^{p} \in R_{+}^{n^{p}}} \phi^{p, n}\left(w^{p}, v, x^{n}, y^{n}\right) \rightarrow \max _{v \in Y} \min _{\left\|w^{p}\right\|=1, w^{p} \in R_{+}^{n^{p}}} \phi^{p}\left(w^{p}, v, x, y\right) .
$$

Then

$$
\max _{p \in \mathcal{P}} \max _{v \in Y} \min _{\left\|w^{p}\right\|=1, w^{p} \in R_{+}^{n^{p}}} \phi^{p, n}\left(w^{p}, v, x^{n}, y^{n}\right) \rightarrow \max _{p \in \mathcal{P}} \max _{v \in Y} \min _{\left\|w^{p}\right\|=1, w^{p} \in R_{+}^{n^{p}}} \phi^{p}\left(w^{p}, v, x, y\right) .
$$


To sum up,

$$
\begin{aligned}
& R\left(F^{n}, x^{n}, y^{n}\right) \\
& \quad=\max _{p \in \mathcal{P}} \max _{u^{p} \in X^{p}} \varphi^{p, n}\left(u, x^{n}, y^{n}\right)+\max _{p \in \mathcal{P}} \max _{v \in Y} \min _{\left\|w^{p}\right\|=1, w^{p} \in R_{+}^{n^{p}}} \phi^{p, n}\left(w^{p}, v, x^{n}, y^{n}\right) \\
& \quad \rightarrow \max _{p \in \mathcal{P}} \max _{u^{p} \in X^{p}} \varphi^{p}(u, x, y)+\max _{p \in \mathcal{P}} \max _{v \in Y} \min _{\left\|w^{p}\right\|=1, w^{p} \in R_{+}^{n^{p}}} \phi^{p}(w, v, x, y) \\
& \quad=R(F, x, y) .
\end{aligned}
$$

Remark 4.1 For any $\varepsilon \geq 0$ and $F \in \mathcal{F}$, we define the set of $\varepsilon$-equilibria at $F$ by $E_{N S}(F, \varepsilon)=$ $\{(x, y) \in f(F): R(F, x, y) \leq \varepsilon\}$, it describes the bounded rationality in population games with uncertain parameters. Especially, by Lemma $4.3, E_{N S}(F, \varepsilon)=E_{N S}(F, 0)$ is the set of NS equilibria for $F$ as $\varepsilon=0$, which describes the complete rationality in population games with uncertain parameters.

Remark 4.2 $M_{1}=(\mathcal{F}, X, Y, T, R)$ is the model of bounded rationality for population games with uncertain parameters. Similar to Definition 4.1 and Definition 4.2, we can define the model $M_{1}$ to be structurally stable and robust to $\varepsilon$-equilibria at $F \in \mathcal{F}$, here we will not go into details.

Now we give the following stability results.

Theorem 4.1 If $M_{1}=(\mathcal{F}, X, Y, T, R)$ is the model of bounded rationality for population games with uncertain parameters we mentioned above, then all stability results of Lemma 4.1 are applicable for it.

Proof From our discussions above, $(\mathcal{F}, \rho)$ is complete, $X \times Y$ is compact, $f: \mathcal{F} \rightarrow X \times Y$ and $R: \mathcal{F} \times X \times Y \rightarrow R_{+}$are continuous, so Lemma 4.1 holds for $M_{1}$.

\section{Conclusion}

In this paper, we proved the existence theorem of NS equilibria for population games with uncertain parameters. Meanwhile, some stability results of NS equilibria have been obtained by establishing a model of bounded rationality. Due to the uncertainty of decisionmaking environment for game problems, the population games with uncertain parameters expand the application scope of classical population games, so they are more practical and have a more popularly theoretic value. However, the paper only focuses on the equilibria for single-objective population games with uncertain parameters, the equilibria for multiobjective ones will be the next research direction in the future.

Acknowledgements

The authors would like to thank the editors and reviewers in helping improvement of the manuscript.

Funding

This work is supported by National Natural Science Foundation of China $(11271098,11761023,11861020)$ and Science and Technology Fund of Guizhou Province (No. [2019]1067). 


\section{Competing interests}

The authors declare that they have no competing interests.

\section{Authors' contributions}

All authors contributed equally to this work. All authors read and approved the final manuscript.

\section{Author details}

${ }^{1}$ School of Mathematics and Statistics, Guizhou University, 550025, Guiyang, Guizhou, China. ²Department of

Mathematics and Computer, Guizhou Normal College, 550018, Guiyang, Guizhou, China.

\section{Publisher's Note}

Springer Nature remains neutral with regard to jurisdictional claims in published maps and institutional affiliations.

\section{Received: 2 September 2020 Accepted: 30 December 2020 Published online: 19 January 2021}

\section{References}

1. Nash, J.: Noncooperative Games. Dissertation, University of Princeton (1950)

2. Hofbauer, J., Sandholm, W.H.: Stable games and their dynamics. J. Econ. Theory 144, 1665-1693 (2009)

3. Sandholm, W.H.: Population Games and Evolutionary Dynamics. MIT Press, Cambridge (2011)

4. Sandholm, W.H.: Large population potential games. J. Econ. Theory 144, 1710-1725 (2009)

5. Lahkar, R., Sandholm, W.H.: The projection dynamic and the geometry of population games. Games Econ. Behav. 64, 565-590 (2008)

6. Reluga, T.C., Galvani, A.P.: A general approach for population games with application to vaccination. Math. Biosci. 230 67-78 (2011)

7. Yang, G.H., Yang, H.: Stability of weakly Pareto-Nash equilibria and Pareto-Nash equilibria for multiobjective population games. Set-Valued Var. Anal. 25, 427-439 (2017)

8. Yang, G.H., Yang, H., Song, Q.Q.: Stability of weighted Nash equilibrium for multiobjective population games. J. Nonlinear Sci. Appl. 9, 4167-4176 (2016)

9. Yang, Z., Zhang, H.Q.: Essential stability of cooperative equilibria for population games. Optim. Lett. 13, 1573-1582 (2019)

10. Anderlini, L., Canning, D.: Structural stability implies robustness to bounded rationality. J. Econ. Theory 101, 395-422 (2001)

11. Yu, C., Yu, J.: On structural stability and robustness to bounded rationality. Nonlinear Anal. TMA 65, 583-592 (2006)

12. Yu, C., Yu, J.: Bounded rationality in multiobjective games. Nonlinear Anal. TMA 67, 930-937 (2007)

13. Yu, J., Yang, H., Yu, C.: Structural stability and robustness to bounded rationality for non-compact cases. J. Glob. Optim. 44, 149-157 (2009)

14. Yu, J., Yang, Z., Wang, N.F.: Further results on structural stability and robustness to bounded rationality. J. Math. Econ. 67, 49-53 (2016)

15. Harsanyi, J.C.: Games with incomplete information played by "Bayesian" players: part I. Manag. Sci. 14, 159-182 (1968)

16. Larbani, M.: Non-cooperative fuzzy games in normal form: a survey. Fuzzy Sets Syst. 160, 3184-3210 (2009)

17. Wang, C., Tang, W.S., Zhao, R.Q.: Static Bayesian games with finite fuzzy types and the existence of equilibrium. Inf. Sci. $178,4688-4698$ (2008)

18. Zhukovskii, V.I.: Linear Quadratic Differential Games. Naoukova Doumka, Kiew (1994)

19. Larbani, M.: About the existence of Nash-Slater equilibrium for a non-cooperative game under uncertainty. In: Caballero, R., Steuer, R. (eds.) Advances in Multiple Objective and Goal Programming, vol. 455, pp. 255-262. Springer, Berlin (1997)

20. Larbani, M., Lebbah, H.: A concept of equilibrium for a game under uncertainty. Eur. J. Oper. Res. 117, 145-156 (1999)

21. Farida, A., Abdelkader, M.: Solution concept for a two person bargaining problem with unknown parameters. Int. J. Uncertain. Fuzziness Knowl.-Based Syst. 19, 39-49 (2011)

22. Ansari, Q.H., Schaible, S., Yao, J.C.: The system of generalized vector equilibrium problems with applications. J. Glob. Optim. 22, 3-16 (2002)

23. Klein, E., Thompson, A.C.: Theory of Correspondences. Wiley, New York (1984)

24. Fan, K.: Fixed point and minimax theorems in locally convex topological linear spaces. Proc. Natl. Acad. Sci. USA 38, 121-126 (1952)

25. Glicksberg, I.L.: A further generalization of the Kakutani fixed point theorem, with applications to Nash equilibrium points. Proc. Am. Math. Soc. 3, 170-174 (1952)

26. Yu, J.: The Continued Study on Game Theory and Nonlinear Analysis. Science Press, Beijing (2011) 